\title{
Correction to: Dielectric and magnetic properties of cobalt doped $\gamma-\mathrm{Fe}_{2} \mathrm{O}_{3}$ nanoparticles
}

\author{
Muhammad Hussain ${ }^{1}$ - Rajwali Khan ${ }^{1} \cdot$ Zulfiqar $^{1} \cdot$ Tahir Zeb Khan $^{1}$ - Gulzar Khan ${ }^{1}$. Shaukat Khattak ${ }^{1}$. \\ Muneeb Ur Rahman ${ }^{2} \cdot$ Shahid Ali ${ }^{3} \cdot$ Zainab Iqbal $^{1} \cdot$ Burhanullah $^{2} \cdot$ Kashif Safeen $^{1}$
}

Published online: 20 December 2019

๑) Springer Science+Business Media, LLC, part of Springer Nature 2019

\section{Correction to: Journal of Materials Science: Materials in Electronics (2019) 30:13698-13707 https://doi.org/10.1007/s10854-019-01747-6}

The original version of this article unfortunately published with few errors in Fig. 1 and Table 1 which was reported to the Editorial Office. This has been corrected by publishing this Erratum.

The correct form of this Fig. 1 and Table 1 is as follows:

Figure 1 in the published manuscript seems to be having made by the hand but in reality, since the fact that whenever $\mathrm{X}$-ray source, Cuk $\alpha$, is used for iron oxide, the fluorescence takes place and consequently, polychromatic radiations are emitted. The results in irregular and noisy peaks as evident in the below image (Fig. 1) can be resolved by choosing an element for the tube that is appropriate to the studied sample or experimental requirement, and Co for samples with $\mathrm{Fe}$ is the most appropriate option which is not available here. To remove the background noise, the original image was therefore made smooth in the published manuscript. Besides, the XRD results are also in agreement with the FTIR (Fig. 2), Raman (Fig. 4) and TEM (Fig. 5) results.

There is an error in the indexation in Fig. 1, Table 1 and text: the first peak at about $30^{\circ}$ is 200 instead of 220 .

The original article can be found online at https://doi.org/10.1007/ s10854-019-01747-6.

Zulfiqar

sydzlfqr@zju.edu.cn; sydzlfqr@awkum.edu.pk

1 Department of Physics, Abdul Wali Khan University, Mardan, KP 23200, Pakistan

2 Department of Physics, Islamia College, Peshawar, KP 25120, Pakistan

3 Department of Physics, University of Peshawar, Peshawar, KP 25120, Pakistan

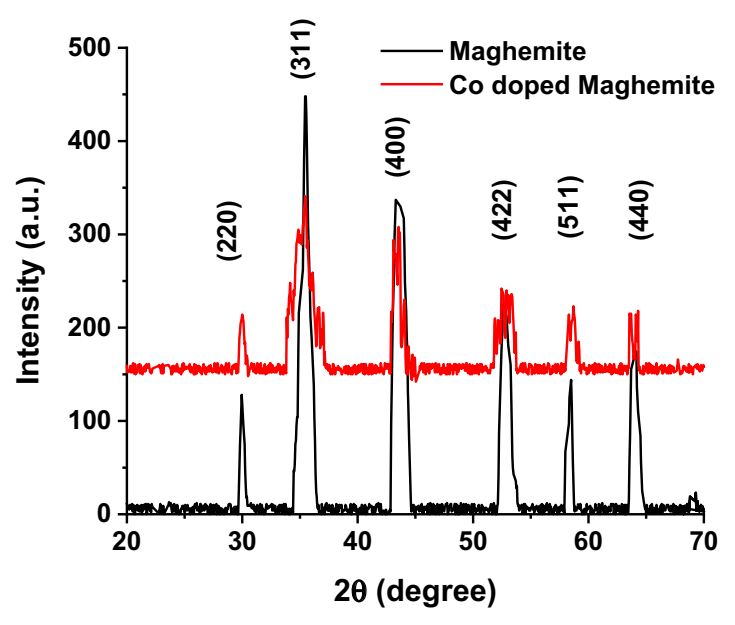

Fig. 1 XRD peaks an of un-doped maghemite and Cobalt doped maghemite

Therefore, the indexation of the peak in Fig. 1, Table 1 and text at $30^{\circ}$ should be read as (220) rather than (200).

The lattice parameters in Table 1 for both pure and doped samples have been calculated for all their corresponding three most intense peaks which are the same up to two decimal numbers, they have therefore been mentioned only for the first peak. The lattice parameters for all three peaks are given in Table 1. While looking at the porosity of the samples in Table 1 of the published paper one wonders how the porosity of the powder has been calculated. This is clarified here that the porosity has been calculated when the samples were in their pellet form rather than in powder form. A similar procedure has also been followed in the Ref. [1].

Since the peaks were of irregular shape, we fitted the peaks and calculated the crystallite size. The values of crystallite size are corrected as given below in Table 1 . 
Table 1 Different parameters of XRD patterns

\begin{tabular}{|c|c|c|c|c|c|c|c|c|}
\hline \multirow{2}{*}{$\frac{\text { Properties }}{\mathrm{hkl}}$} & \multicolumn{4}{|c|}{ Un-doped $\gamma-\mathrm{Fe}_{2} \mathrm{O}_{3}$} & \multicolumn{4}{|c|}{ Cobalt doped $\gamma-\mathrm{Fe}_{2} \mathrm{O}_{3}$} \\
\hline & 200 & 311 & 400 & 422 & 200 & 311 & 400 & 422 \\
\hline $\operatorname{Dhkl}(\AA)$ & 2.9 & 2.5 & 2.1 & 1.7 & 3.0 & 2.5 & 2.1 & 1.7 \\
\hline Crystallite size. $\mathrm{t}(\mathrm{nm})$ & 11.2 & 8.0 & 8.4 & 10.2 & 20.9 & 10.7 & 8.9 & 20.6 \\
\hline Average crystallite size $\bar{t} \pm \sigma(\mathrm{nm})$ & $9 \pm 1$ & & & & $15 \pm 6$ & & & \\
\hline Lattice parameter a $(\AA)$ & 8.4 & & & & 8.454 & & & \\
\hline Lattice volume $\mathrm{V}(\AA)^{3}$ & 587.9 & & & & 604.3 & & & \\
\hline Sample density $\rho(M)=\frac{\mathrm{m}}{\pi r^{2} \mathrm{~h}}\left(\mathrm{~g} / \mathrm{cm}^{3}\right)$ & 1.42 & & & & 1.27 & & & \\
\hline X-rays density $\rho(\mathrm{X})=\frac{8 \mathrm{M}}{N_{\mathrm{A}} a^{3}}\left(\mathrm{~g} / \mathrm{cm}^{3}\right)$ & 3.62 & & & & 3.46 & & & \\
\hline $\mathrm{P}=$ Porosity $=1-\frac{\rho(\mathrm{M})}{\rho(\mathrm{X})}$ & 0.6 & & & & 0.6 & & & \\
\hline$A=$ surface area $=\frac{6}{t_{\rho}(X)}\left(\mathrm{cm}^{2} / \mathrm{g}\right)$ & $3.4 \times 10^{-6}$ & & & & $1.1 \times 10^{-6}$ & & & \\
\hline
\end{tabular}

\section{Reference}

1. I.H. Gul, F. Amin, A.Z. Abbasi, M. Anis-ur-Rehman, A. Maqsood, Physical and magnetic characterization of co-precipitated nanosize Co-Ni ferrites. Scr. Mater. 56, 497-500 (2007)
Publisher's Note Springer Nature remains neutral with regard to jurisdictional claims in published maps and institutional affiliations. 